\title{
Experimental Evaluation of an Integrated Datalink and Automation-Based Strategic Trajectory Concept
}

\author{
Eric Mueller* \\ NASA Ames Research Center, Moffett Field, CA 94035
}

\begin{abstract}
This paper presents research on the interoperability of trajectory-based automation concepts and technologies with modern Flight Management Systems and datalink communication available on many of today's commercial aircraft. A tight integration of trajectory-based ground automation systems with the aircraft Flight Management System through datalink will enable mid-term and far-term benefits from trajectory-based automation methods. A two-way datalink connection between the trajectory-based automation resident in the Center/TRACON Automation System and the Future Air Navigation System-1 integrated FMS/datalink in NASA Ames' B747-400 Level D simulator has been established and extensive simulation of the use of datalink messages to generate strategic trajectories completed. A strategic trajectory is defined as an aircraft deviation needed to solve a conflict or honor a route request and then merge the aircraft back to its nominal preferred trajectory using a single continuous trajectory clearance. Engineers on the "ground side" of the datalink generated lateral and vertical trajectory clearances and transmitted them to the Flight Management System of the 747; the airborne automation then flew the new trajectory without human intervention, requiring the flight crew only to review and to accept the trajectory. This simulation established the protocols needed for a significant majority of the trajectory change types required to solve a traffic conflict or deviate around weather. This demonstration provides a basis for understanding the requirements for integration of trajectory-based automation with current Flight Management Systems and datalink to support future National Airspace System operations.
\end{abstract}

\section{Nomenclature}

$\begin{array}{ll}A C A R S & =\text { Aircraft Communication Addressing and Reporting System } \\ A T C & =\text { Air Traffic Control } \\ \text { ATSP } & =\text { Air Traffic Service Provider } \\ C P D L C & =\text { Controller-Pilot Datalink Communications } \\ C T A S & =\text { Center/TRACON Automation System } \\ C V S R F & =\text { Crew Vehicle Systems Research Facility } \\ F A N S & =\text { Future Air Navigation System } \\ F M S & =\text { Flight Management System } \\ J P D O & =\text { Joint Planning and Development Office } \\ N A S & =\text { National Airspace System } \\ N G A T S & =\text { Next Generation Air Transportation System } \\ R N P & =\text { Required Navigation Performance } \\ T B O & =\text { Trajectory-based Operations } \\ V D L & =\text { VHF Data Link }\end{array}$

\section{Introduction}

$\mathrm{T}$ he concept of operations for the Next Generation Air Transportation System (NextGen) relies heavily on the exchange of 4D trajectory information to achieve higher levels of automation for conflict detection and

\footnotetext{
*Aerospace Engineer, Automation Concepts Research Branch, M/S 210-10, AIAA Member
} 
resolution, metering and trajectory changes while operating under conditions of increased air traffic density, reduced allowable accident rates, lower controller workload, and greater accommodation of user preferences. ${ }^{1}$ In accordance with guidance from the multi-agency Joint Planning and Development Office (JPDO) that "it's all about the users," the goal of this research is to accommodate, to the maximum extent possible, the preferences of the airspace users, and to minimize, within strict safety standards, the adverse impact of Air Traffic Control (ATC) initiatives and restrictions. The users are generically defined as any agent that makes use of ATC services and are most commonly represented by airlines and business aviation groups. It is proposed that the preferences of these users can be better accommodated within the constraints of the ATC system by moving from today's tactical, voice-based clearances to the use of continuous trajectories transmitted over datalink.

Previous research has shown the feasibility and benefit of integrating ground automation and the airborne Flight Management Systems (FMS) through datalink communication, but not all aspects of such an integration have been satisfactorily resolved. Basic exchanges so far demonstrated include Transfer of Communications (TOC), altimeter calibration data, and direct-to messages. ${ }^{7}$ Corresponding demonstrations of complex clearances with real automation systems are not complete. These complex clearances, which form the foundation of Trajectory-Based Operations (TBO), are defined as arbitrary route and/or altitude changes; the amended route needs not pass through named waypoints but may instead be specified with latitude and longitude coordinates. Important issues concerning the implementation of such trajectories remain to be resolved: the respective roles and responsibilities of pilots and controllers; the degree of flight deck automation required to limit workload increases; the precision with which aircraft would be able to follow 4D clearances; and the expected impact of changes, upgrades or modifications to existing aircraft equipment. ${ }^{2,3}$ Several concepts have been developed that integrate air and ground automation with datalink to provide specific answers to these questions. A European experiment uploaded Required Time of Arrival (RTA) clearances to aircraft at takeoff and measured the accuracy with which the airborne FMS was able to meet that RTA at the runway threshold. ${ }^{4}$ This example of time-based air traffic management (ATM) demonstrated one element of TBO by showing that aircraft automation is able to accurately meet an RTA over a multi-hour time horizon in real-world, low aircraft-density conditions. Further work will expose modifications to the concept necessary to apply it under denser air traffic scenarios and accommodate the trajectory uncertainty inherent to RTA clearances.

The work described in this paper has two objectives: to determine the degree to which current air and ground automation capabilities together with datalink support trajectory-based operations; and to present simulation results that validate the feasibility of conducting TBO in today's environment. These objectives are met using simulations that connect a prototype ground-based automation system, the Center/TRACON Automation System, ${ }^{5}$ with the most advanced airborne automation currently flying, the Future Air Navigation System-1 (FANS1) on a 747-400 level D simulator at NASA Ames Research Center. ${ }^{6}$ The simulated datalink between these systems is based on the protocols developed for Controller-Pilot Data Link Communications (CPDLC). This paper describes the strategic trajectory concept in detail, outlines the experimental approach to assessing the feasibility of that concept, and presents results on the suitability of current automation systems for TBO and the feasibility of those operations in today's air traffic system.

\section{Background}

A number of organizations have examined many aspects of TBO through research and simulation, but a full evaluation of the end-to-end concept with all operational elements (flight deck hardware, datalink infrastructure, etc.) has not been conducted. Researchers have proposed concepts for integrating the User Request Evaluation Tool (URET) with datalink to achieve TBO and significant benefits for airspace users; ${ }^{7,8}$ but because this work is ongoing, the fundamental barrier to trajectory-based operations is not yet known. Early studies of the concept have shown that aircraft trajectories may be predicted by ground based automation systems with an accuracy of tens of seconds over a 20 minute horizon. ${ }^{9,10}$ They also identified many of the problems still suspected of being the primary obstacles to the introduction of TBO: unmodeled wind errors; quality of surveillance data; commonality of flight crew procedures among different airlines and aircraft types; and the ability to certify datalink communications to an equivalent level of safety as voice. ${ }^{11}$ Since then, several groups have quantified the magnitude of these problems and suggested the most important are safety and procedural commonality, assuming a number of technical and cost problems are resolved. ${ }^{12,13,14}$ High fidelity simulations with controllers and pilots confirm the need to resolve technical issues like the maximum allowable pilot response time to a datalink message, ${ }^{15}$ and additional evaluations with actual flight hardware would provide valuable data. 
One of the most important challenges facing TBO is implementing strategic clearances on the flight deck using current FMS and datalink equipment. While the problem has not been fully investigated, it is known that integrated FMS and datalink operations cannot replace voice using current day procedures. ${ }^{16}$ Datalink is a fundamentally different means of communication with different strengths and weaknesses, so it will always compare poorly if datalink is introduced in direct substitution under current operations. ${ }^{17,18}$ A large body of research does exist that explores better ways of interfacing the flight crew with datalink operations in a way that captures the unique benefits of such a medium, and aspects of those studies helped inform this research project. ${ }^{19,20}$

Modeling and simulation studies also suggest that ground based automation provides significant benefit in terms of safety, capacity and efficiency and can reduce controller workload if procedures are structured correctly. ${ }^{21}$ The challenge is to integrate ground based automation with FMS and datalink to capture all the benefits cited in literature without an unacceptable increase in pilot and controller workload. For instance, $20 \%$ of the 58 operational errors analyzed in a recent study were the direct result of misunderstanding voice transmissions between the flight crew and controller (which datalink would remove), and 52\% were from improperly analyzed horizontal or vertical clearances, which should be caught by the ground automation's trajectory prediction engine. ${ }^{22}$ Hasan et. al. forecast up to half a billion dollars a year in savings from trajectory negotiation over datalink alone.

Researchers hypothesize that benefits will be realized after implementation of datalink in the National Airspace System (NAS). The introduction of the experimental Controller-Pilot Data Link Communications (CPDLC) project in Miami represented the first large scale experiment with datalink in the US and spurred several studies touting the benefits of such a system. Because the CPDLC message set emulates voice-based clearances, it does not take full advantage of the increase in message complexity afforded by electronic communications. As a result, expectations for benefits from adoption of CPDLC tend to be conservative. ${ }^{7}$ Those benefits include reduction in voice frequency congestion, which is currently driving Europe to adopt datalink more quickly than the US, transfer of communications capability, and basic information sharing like altimeter settings. ${ }^{23}$ Some estimates indicate that the cost of CPDLC implementation can be covered through these conservative benefit mechanisms by reducing flight times more than 9400 minutes per day within 15 years. ${ }^{24}$ Trajectory-based operations will be able to leverage this datalink infrastructure once it is brought back into service.

\section{Strategic Trajectory Operations and Clearances}

This section describes the concept for strategic trajectory operations as tested in simulation. Strategic trajectory clearances deviate an aircraft to solve a problem or honor a user request and then return it to its nominal preferred trajectory using a single, continuous clearance. Examples of such horizontal and vertical strategic trajectory

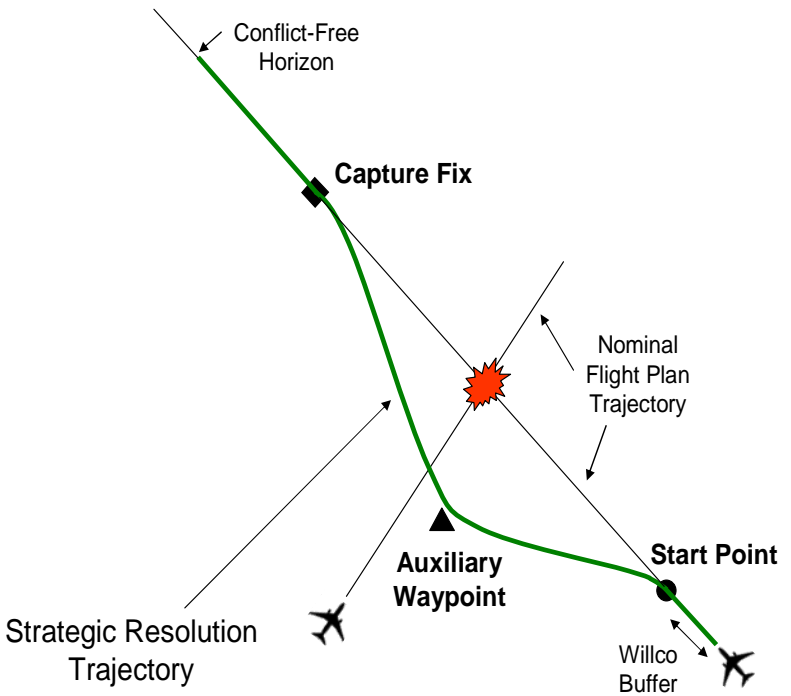

Figure 1. Single auxiliary waypoint horizontal trajectory clearance. clearances are shown in Figure 1 and Figure 2. In all cases, problems are solved using a continuous trajectory that includes a start point, at least one intermediate point specific to the problem being solved, and a capture point that returns the aircraft back to its original trajectory profile. It is expected that such trajectories: 1) will reduce both controller and pilot workload by solving problems with a single strategic clearance instead of multiple tactical clearances as in today's operations; 2) will improve overall predictability in the NAS since the complete trajectory for each aircraft is always known; 3 ) are well suited for FMS implementation and datalink communication; 4) are compatible with the simultaneous solution of conflict and time-based metering problems, which are common in complex, high density airspace. Laboratory research and limited simulation and operational testing suggest these characteristics will lead to fewer restrictions, lower workload and increased airspace capacity and efficiency. ${ }^{25,26,27}$ 


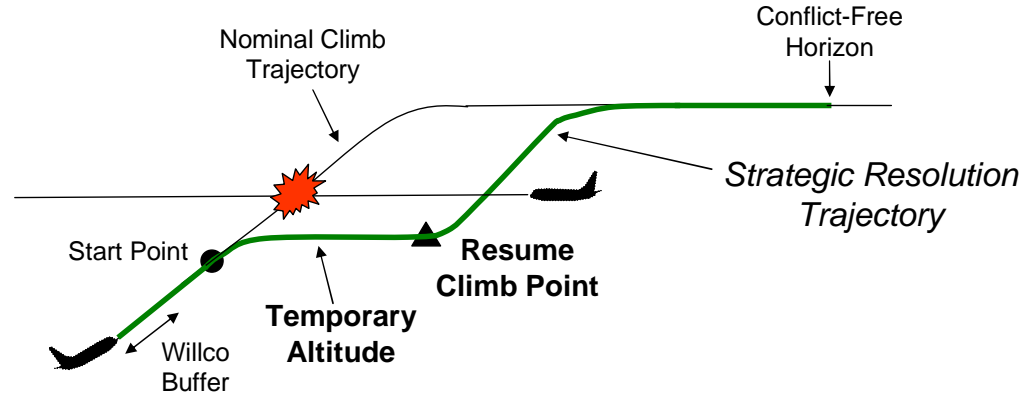

Figure 2. Interrupted climb clearance.
Trajectory changes may be initiated and exchanged in two ways: clearances are sent from a central service provider (e.g., ground automation or a controller using ground automation) to the aircraft; or trajectory change requests are initiated by the flight crew or flight deck automation. The concept tested in this experiment is the former due to the immaturity of operational capabilities for creating, analyzing and transmitting complex trajectory requests from the flight deck; however, the ground-based system is designed to accept trajectory change requests from any source whether or not the flight deck currently has the ability to generate those requests. Concepts for realizing the latter capability will be developed according to the results of the initial experiment.

A prototype trajectory based automation capability has been developed at NASA that can recognize the need for and generate a revised trajectory for conflict resolution and is designed to be directly integrated with the controller's Plan View Display (PVD). If a user provides the trajectory, some provision must be made for specifying, transmitting and displaying that trajectory to the controller. If a controller decides a clearance is required, he uses a trajectory-based "what if" trial-planning capability, also developed at NASA, to design the strategic trajectory. Any of these three methods will be functionally equivalent when presented to the controller, and the same tools will be available to analyze the feasibility of the proposed trajectory no matter how it was originally created (by the automation, user-specified, controller-designed). Once the controller has reviewed the trajectory and modified it to his satisfaction, another automation function transmits the clearance to the appropriate aircraft. That function translates the strategic clearance into CPDLC messages and sends them to the aircraft using a currently-available datalink service (ACARS, VDL, etc.). When the flight crew has received the clearance, they review it up to the allowable WILCO buffer - nominally two minutes but subject to change according to the results of simulation. The near-term horizon of this experiment suggests that significant review and modification of the trajectory by the flight deck is infeasible because of the lack of a traffic information display, but this is another research question that the simulations will help to answer.

The basic trajectory clearance classes, horizontal and vertical, are shown in Figure 1 and Figure 2, but for simulation and operational implementation these classes have to be broken down into specific applications for each strategic clearance. These applications are mentioned above and include metering (see example in Figure 3), direct routing (Figure 4), and user-preferred routes (Figure 5 and Figure 6). The next stage is to map each trajectory clearance onto the available set of datalink messages so that such clearances, whether generated by a controller, flight deck, or automation system, can be efficiently transmitted across datalink to the appropriate parties. Most of the necessary clearances for this concept may be implemented with currently-available messages, as will be shown in Section V, but some will require either defined, but not yet implemented messages, or entirely new messages.

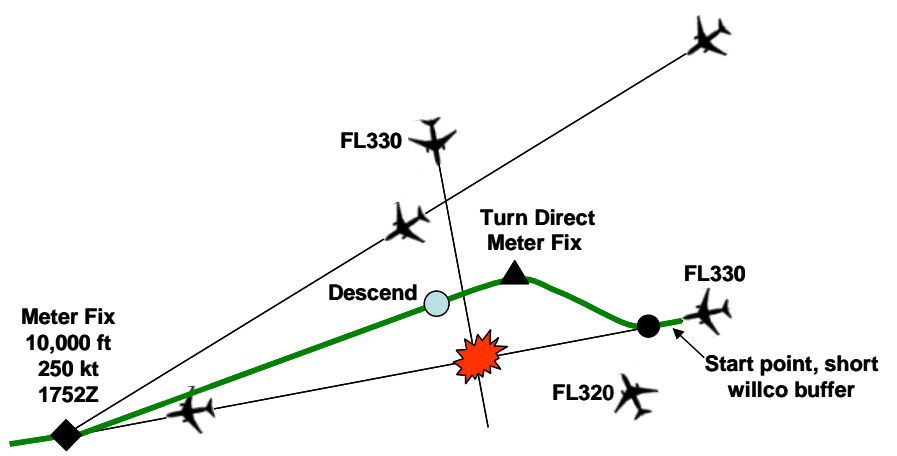

Figure 3. Strategic metering clearance with resolution in cruise and descent.

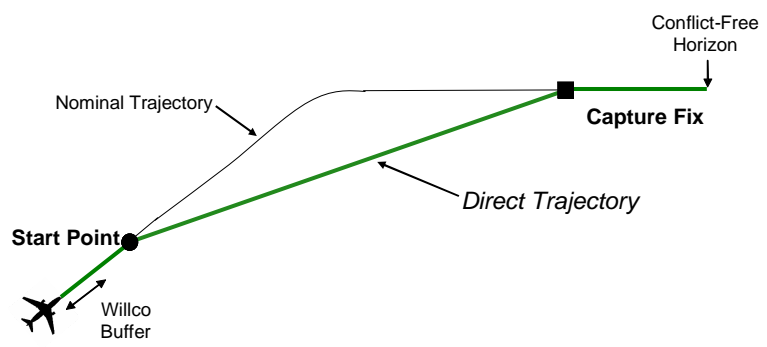

conflict Figure 4. Direct-to strategic trajectory. This is an example of the type of clearance that could be userinitiated. 


\section{Experimental Approach}

Modeling and real-time simulation activities were designed to test the interoperability of trajectory-based automation and the FANS1 integrated FMS/datalink capability now available on many commercial aircraft. The simulation used the trajectory-based automation functionality and software resident in the Center-TRACON Automation System and a fully certified, Level D 747-400 simulator which is fully equipped with actual FANS1 hardware and software. ${ }^{6}$ The integrated FMS/datalink capability is critical for the early tests because of the expectation that manually entering a continuous strategic trajectory will unduly increase flight deck workload and be prone to errors. A set of draft procedures a flight crew might use to execute a strategic trajectory is given in the Appendix. A real-time software interface was developed to support two-way communication between CTAS and the FANS1 hardware using CPDLC format. From the perspective of the 747 FMS, which is actual flight hardware, the messages received from CTAS via CPDLC data communication protocol are identical to that which it would receive in an operational setting. The objectives of this simulation were to:

- Demonstrate real-time two-way connectivity between CTAS trajectory-based automation and FANS1 integrated FMS/datalink equipment (747-400).

- Investigate datalink communication of a variety of strategic trajectory clearances to the FANS1 FMS via CPDLC protocol.

- Determine which CPDLC messages are most efficient for implementation of several different types of strategic clearances in a 747-400 FMS (horizontal, vertical, metering).

- Confirm messages could be loaded by the FMS, and confirm the clearance could be flown and that its performance was consistent with the original intentions of the trajectory-based automation system.

- Measure the accuracy of CTAS trajectory predictions with respect to the simulator trajectory as flown by the FMS and the autopilot.

- Create the capability to display a user request on the controller display in CTAS.

The specification of CTAS trajectory clearances into FMS-readable messages involved converting the CTAS trajectory format into a sequence of CPDLC messages, populating the appropriate Abstract Syntax Notation (ASN1) format data structures and encoding that data according to Packed Encoding Rules for datalink communication to

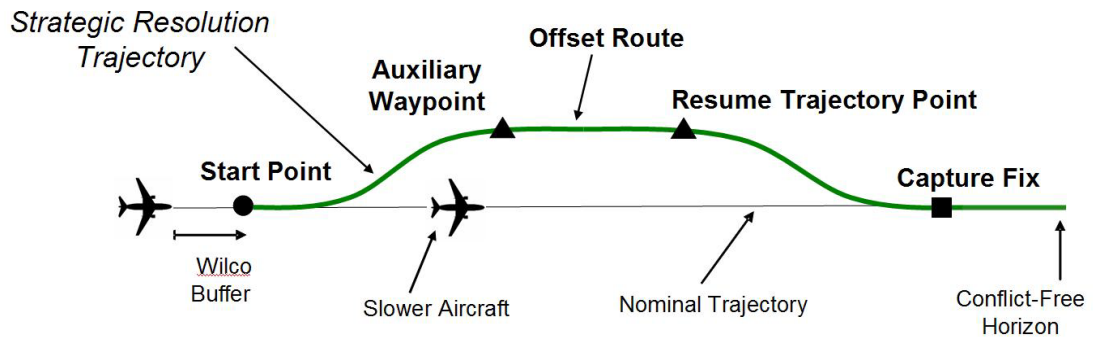

Figure 5. Offset trajectory for overtaking a slower aircraft. This is also a potentially user-initiated clearance.

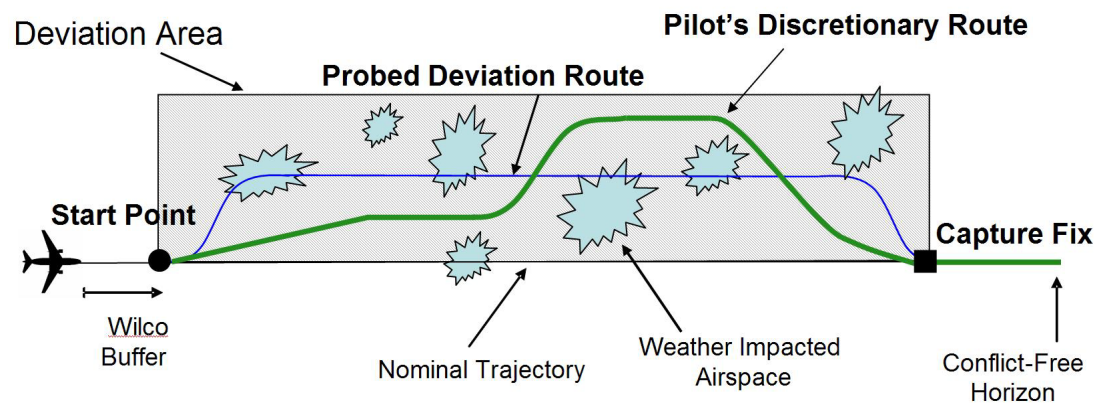

Figure 6. Example of a user-initiated deviation request. The aircraft is free to deviate up to a specified distance from the nominal route, in this case to avoid weather. This trajectory was not tested in simulation. the aircraft. Several different sequences of message options were selected that could define a specific trajectory and the performance of each evaluated for its interoperability with the FMS. Results suggested the best approaches for implementing each of the trajectory clearance types (horizontal, vertical, metering). In some trajectory cases, particularly those with altitude changes in cruise, the most efficient implementation was to concatenate more than one message element for a given clearance: a lateral trajectory change and a manually executed CLIMB TO AND MAINTAIN or DESCEND TO AND MAINTAIN message element. Also measured was the accuracy of the CTAS trajectory predictor compared with the aircraft dynamic model contained in the simulator. These measurements suggested ways to improve the design of the ground 
automation trajectory predictor and helped formulate requirements for accurate modeling of TBO, for example the specification of a WILCO buffer time. The metrics used to evaluate the performance of the concept in this simulation are:

- $\quad$ Ability to send CTAS clearance to 747-400 FMS.

- Number of ways to define a CTAS clearance in CPDLC messages.

- Number of concatenated messages required to specify a single trajectory clearance.

- Accuracy of flown trajectory compared with that specified by clearance.

- Accuracy of CTAS trajectory prediction compared with flown trajectory.

- Ability of aircraft to stay in its preferred performance envelope while executing clearances.

- Effect of weather data (winds) on accuracy of trajectory prediction.

\section{Results}

The error in the ground system prediction of an aircraft's trajectory derives from two sources: the error in the ground automation prediction of the trajectory; and the error between that trajectory specification in CPDLC and the actual aircraft trajectory. The first result below addresses the latter of these error sources and shows that a single CPDLC message is suitable for all the trajectory types tested here. The second section reports the accuracy of the prediction with respect to the flown trajectory. The last section gives a preliminary indication of the minimum time a crew would require to execute a strategic clearance.

\section{A. CPDLC Suitability for Strategic Trajectory Specification}

A basic question to be answered by this simulation was the extent to which current systems support the vision of strategic trajectory operations in the NAS, particularly the datalink format CPDLC. Because CPDLC was designed to pass messages replacing the voice-based tactical clearances (e.g., climb to and maintain..., turn left bearing...), it was not clear that it would be suitable for defining strategic trajectories. The simulations showed that CPDLC is capable of specifying all the types of strategic trajectories discussed above (see Figure 1 through Figure 5) using only one or two message elements, not including the pilot-initiated (user-initiated) deviation request (Figure 6), which was not tested. For example, if an aircraft in cruise has the following flight plan and CLARE is the next active waypoint:

CVE . . . CLARE . POTEN . .TEKBE . . BEKEN . . SWB . MCB . . ATL

and the controller would like to vector the aircraft to an auxiliary waypoint to the south before returning the aircraft to a downstream waypoint (BEKEN), he may do so by issuing the following clearance via datalink:

um79: CLEARED TO [BEKEN] VIA [EIC262122..EIC252107]

This single clearance is all that is required to fully specify the strategic trajectory; because CPDLC message number 79 is used to make the route modification, the rest of the flight plan is unchanged after the aircraft reaches BEKEN. In the above clearance, the values in brackets represent message-specific information, while the unbracketed text is implied when message 79 is used. The Start Point of the trajectory is defined within the second bracket and is in range/bearing format relative to an adapted fix or waypoint (EIC). The auxiliary waypoint is also defined as a range $(107 \mathrm{nmi})$ and bearing $\left(252^{\circ}\right.$ true $)$ from a known waypoint. The automation may also specify these trajectories in terms of latitude and longitude. A graphic display of an example message is given in Figure 7.

Two other message elements were considered for CPDLC implementation of this and other types of trajectories:

um80: CLEARED [route clearance]

um83: AT [pos] CLEARED [route clearance] 


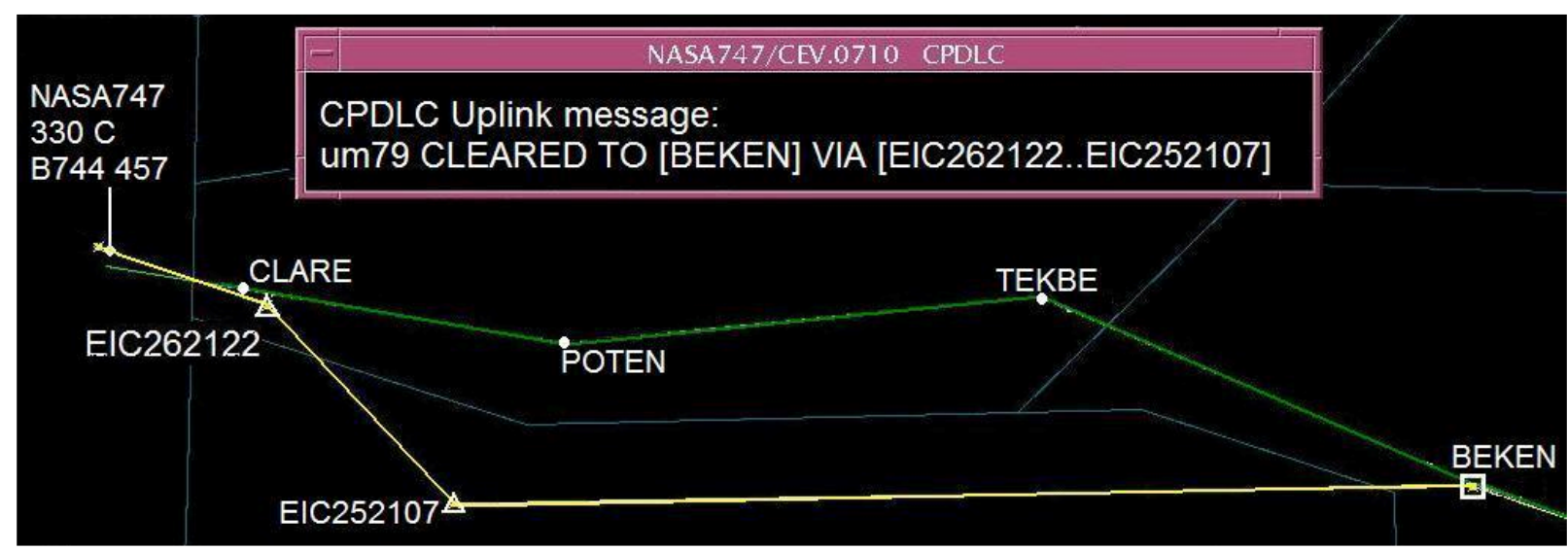

Figure 7. CTAS display of single auxiliary waypoint strategic trajectory in the "trial planning" stage, along with the CPDLC uplink message.

Message 80 is the most general. It replaces the entire flight plan with a new one generated by the ground automation. Message 83 was attractive because it replaces the entire route past a specified waypoint with the new trajectory. It appeared that the Start Point of the trajectory would naturally be that waypoint; however, to avoid flight plan discontinuities the Start Point would have to be a waypoint already specified in the FMS flight plan. That restriction would have unreasonably reduced the flexibility of the concept - flexibility being the primary motivating factor for employing strategic trajectories. In addition, both messages suffered from the same critical problem: the ground automation in every ATC facility cannot be expected to know exactly the flight plan contained in the FMS all the way to the destination. For example, the airline may have a preferred approach procedure at the destination airport that is loaded in the FMS, but which would be erased by the full route uplink. By using um79 the philosophical desire to change only that portion of the trajectory necessary to solve the immediate problem was satisfied, and no additional information sharing requirements were imposed on the concept.

A screen capture of the CTAS display initiating the strategic trajectory is shown in Figure 7. The green (upper) line is the original flight plan while the yellow (lower) line what the controller defined in response to a user request, a traffic conflict, or other air traffic management imperative. This new (yellow) trajectory is known as a "trial plan" and is created by the trajectory-based automation either interactively by the controller or by the automation. In these experiments, the user activates the trial plan trajectory by selecting the flight data block of the appropriate aircraft and then interactively dragging the auxiliary waypoint from the original flight plan to the desired location. When a trial plan is activated CTAS pops up a window, titled "NASA747 CPDLC" in Figure 7, that shows the CPDLC message automatically generated and dynamically updated in response to that particular trial plan. When the controller or test engineer is satisfied with the trial plan, he selects the "Send" button (not shown) and the clearance is transmitted to the 747-400 FMS.

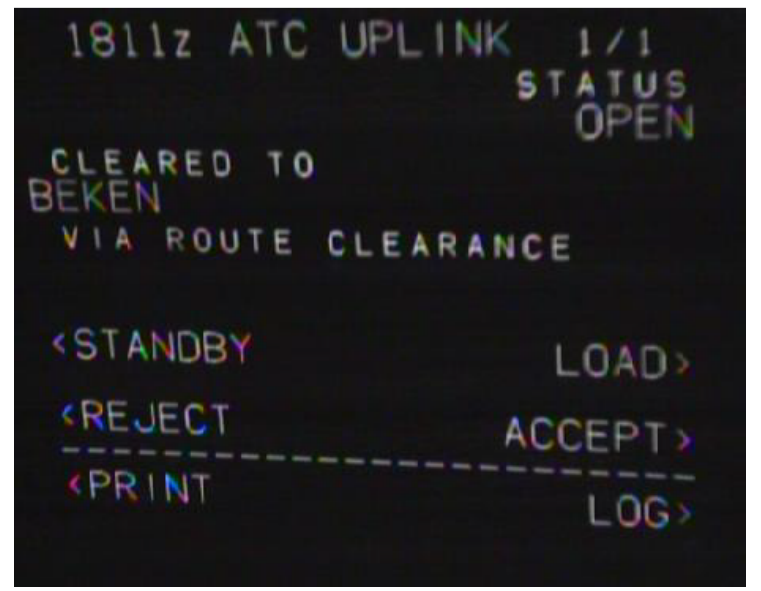

Figure 8. Picture of CDU while flight crew is evaluating the ATC-generated clearance.
When the aircraft receives the ATC message, the flight crew is notified with a chime and a display indicator on the flight deck's Engine Indicator and Crew Alerting System (EICAS). The pilot or co-pilot selects the ATC Message button on the Control Display Unit (CDU) to bring up a textual display of the datalink message. A picture of the CDU showing the received message is given in Figure 8. Only the beginning of the clearance is displayed: CLEARED TO BEKEN VIA ROUTE CLEARANCE. The rest of the clearance is shown graphically on the navigation display after the pilot has selected the LOAD prompt. A screen capture of the navigation display with the new trajectory loaded is shown in Figure 9. If the flight crew is satisfied with the new trajectory they may ACCEPT the clearance, which transfers it to the FMS, and then select EXECUTE (not shown) to command the aircraft to begin flying the new trajectory. Next, the crew 
is presented with a CDU page allowing them to send a WILCO response to the controller, confirming that they have begun flying the new trajectory. When the WILCO response is received the yellow trial plan trajectory automatically becomes the new flight plan, the trajectory for that aircraft is updated, and the trajectory negotiation process is concluded.

The route amendment specified in the datalink format and the trajectory as flown by the aircraft automation in this scenario are shown in Figure 10. The red clearance waypoints (circles) connected by the green lines represent the datalink message that was computed and transmitted based on the ground trajectory automation system. The tracks are actual aircraft position updates, which reflect the flight trajectory the aircraft simulator flew based on the data link message. Note that the aircraft turns inside

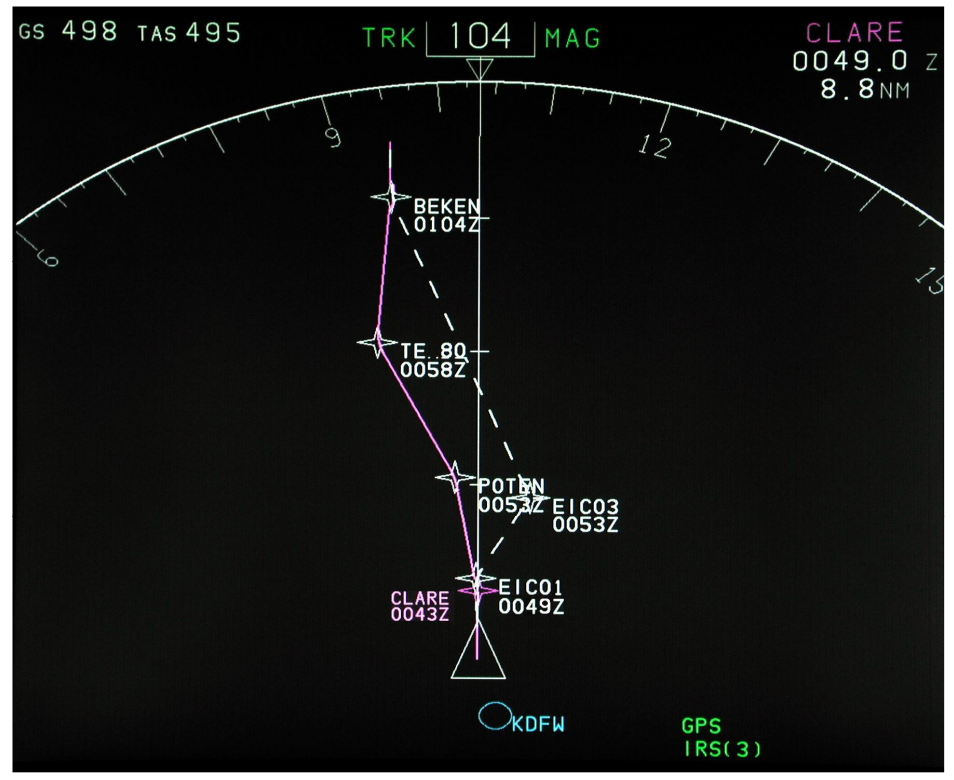

Figure 9. Navigation display of the 747 after the new trajectory has been loaded but not executed.

the auxiliary waypoint as expected. Both the ground and aircraft automation account for flying by the waypoints rather than flying over them, so that source of error will not cause the prediction to be incorrect. The persistent offset from the CPDLC route along the southern East-West leg is likely due to slightly different calculations of the location of the auxiliary waypoint (EIC252107). The bearing from the reference waypoint depends on the magnetic variation in the particular geographic region, and even a fraction of a degree difference over $107 \mathrm{nmi}$ could cause the CPDLC and aircraft trajectories to be offset by $0.5 \mathrm{nmi}$. This problem may be resolved by using the more accurate latitude and longitude specification method; but it is clear that a CPDLC-specified route is consistent with the resulting aircraft trajectory.

The same message element, CPDLC um79, may also be used to define an interrupted climb strategic trajectory (Figure 2). The message consists of a start point along the route of flight, plus an auxiliary waypoint (which may or may not be on the route of flight) with an associated altitude restriction. The next downstream waypoint in the flight plan is where the aircraft reconnects to the original trajectory. For the same scenario as that described above, but with the aircraft climbing from FL180, the appropriate message specifying an interrupted climb at FL270 is simply:

um79: CLEARED TO [BEKEN] VIA EIC262122..EIC252107:270]

The vertical profile of the aircraft as a function of path distance is shown in Figure 11. This behavior is exactly what

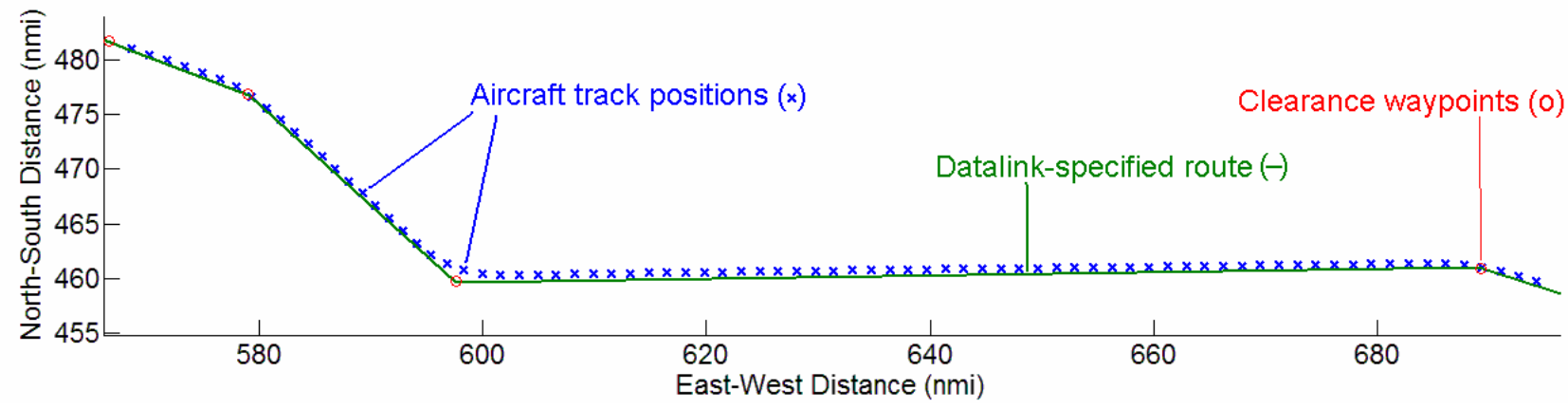

Figure 10. Trajectory flown by the aircraft from Figure 7 vs the route specified in CPDLC format. The mean lateral error is $0.46 \mathrm{nmi}$. with a standard deviation of $0.19 \mathrm{nmi}$. These errors are largely due to the fact that the aircraft turns inside the waypoints and does not fly directly over them. The only data contained in the CPDLC message are the positions of the waypoints drawn as red circles.

8

American Institute of Aeronautics and Astronautics 


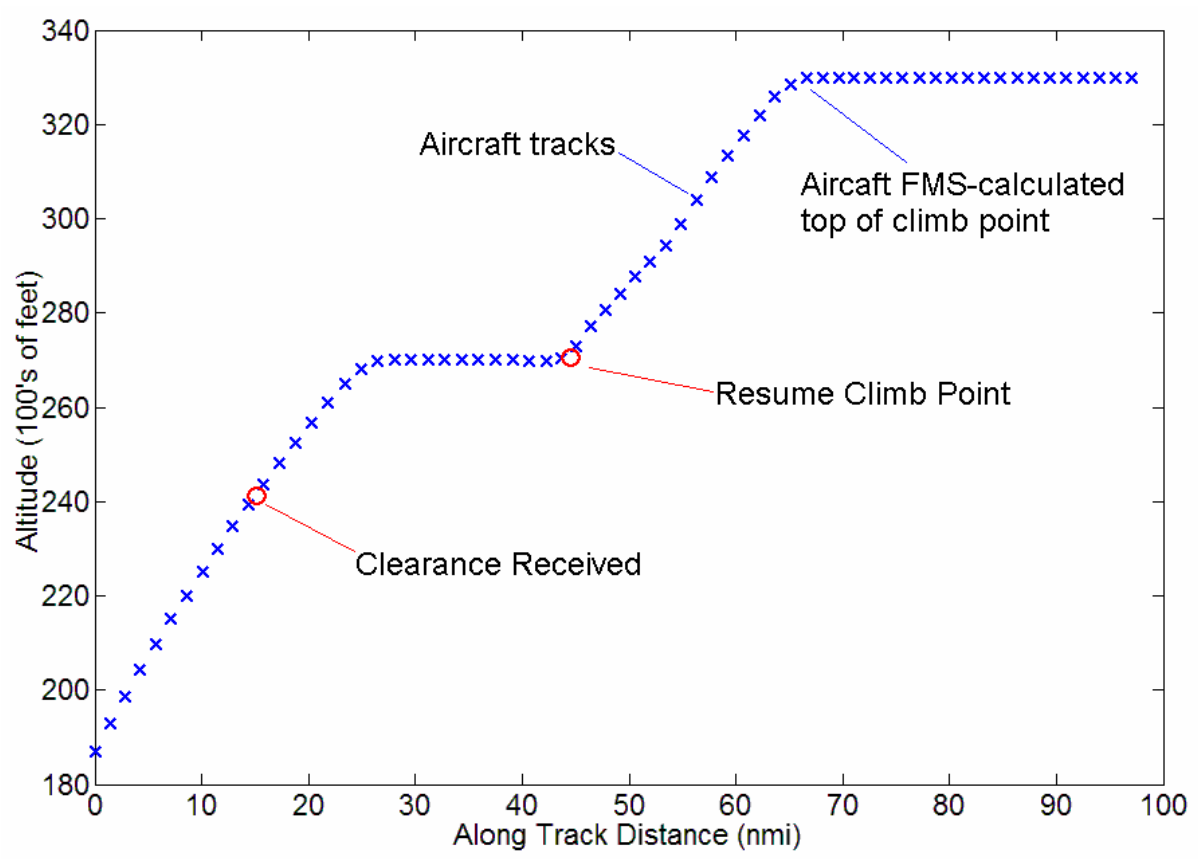

Figure 11. Track data for the interrupted climb clearance. The desired vertical trajectory is shown in Figure 2, and the horizontal trajectory is similar to that in Figure 10. is desired; the aircraft continues climbing at its current rate until it reaches the restriction altitude and maintains that altitude. When the auxiliary waypoint containing the restriction is reached, the aircraft automatically begins climbing again for the cruise altitude with no further action from the flight crew required. The aircraft climbs at its most efficient rate after the resume climb point and is not forced to reach an arbitrary topof-climb point. This automatic execution of the trajectory helps to ensure consistency between the vertical trajectory computed by

the automation to avoid a traffic conflict and the vertical trajectory the aircraft actually flies as it proceeds to its cruise altitude. The resume climb waypoint clearly defines the entire vertical profile from the start point to the cruise altitude. This relieves the controller from having to issue a resume climb command and gives the flight crew a clear indication as to exactly when they will resume their climb. It is also important to note that once the interrupted climb trajectory is accepted by the flight crew (near the clearance received point in Figure 11) it becomes the new nominal trajectory for conflict analysis by the trajectory-based automation system. In addition, if the clearance is only meant to restrict the aircraft from entering the upper regions of airspace, the flight crew is free to shallow the flight path angle and reach the restriction altitude precisely at the resume climb point, which avoids a level trajectory segment. This behavior is consistent with a desire to allow the aircraft maximum flexibility to pursue the most efficient trajectory and potentially saves fuel.

\section{B. Comparison of Flown Trajectory with Predicted Trajectory}

In this section the trajectories flown by the 747-400 simulator are compared to the trajectories generated by the trajectory-based automation upon which the CPDLC data link messages were based. This analysis shows that the essential trajectory information generated by CTAS is faithfully represented in the simplified CPDLC specification. Simulations have been run using the process described in Section V. A and data collected for several trajectory types under several different experimental conditions. Every time a track update is received by CTAS from the simulator, the ground automation generates a new prediction of the trajectory from the current point until the end of the flight plan. This is particularly important when the trajectory changes suddenly because a trial plan was uploaded to the aircraft or after reaching waypoints requiring large changes in heading. Those events tend to create the largest errors in a given scenario so they are particularly important to the prediction process, which will be described below.

The trajectories selected for testing are the most fundamental types among those listed in Section III, and more complicated trajectories may be constructed from them. It is expected that the prediction errors for more complex horizontal trajectories would be of the same order as those reported here, because there is no distinction between CPDLC route messages with different numbers of waypoints from the perspective of the FMS. This generalization may not hold for more complicated vertical clearances, however, and must be investigated further through high fidelity simulations. The trajectories analyzed here are the single auxiliary waypoint clearance, Figure 1, direct-to, Figure 4, and interrupted climb, Figure 2. The clearances were tested in both cruise and climb modes and the alongtrack, cross-track and vertical errors measured between the predicted trajectory and that flown by the 747 simulator. The simulation also analyzed the effects of errors in the ground automation's knowledge of the wind speed and 

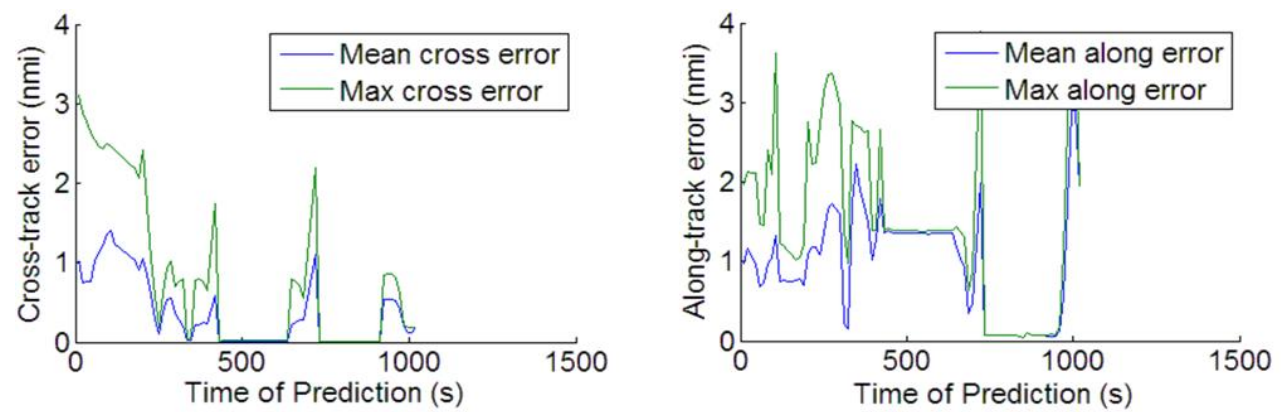

aircraft weight when compared with that in the simulator. Scenarios were run in which the aircraft weight was identical in the two systems and were also run with the actual aircraft weight approximately $20 \%$ lower than that

Figure 12. Along and cross track errors as a function of the time the prediction was made along the flight path. This scenario is for a single auxiliary waypoint clearance (see Figure 1) with no mismatches between simulator and CTAS winds or aircraft weights.

assumed by the ground automation (CTAS). Because CTAS assumes aircraft weight based on the maximum takeoff weight and phase of flight such a mismatch is not uncommon. The wind error, when present, was always set to $10 \mathrm{kt}$ in the easterly direction. It would not have been feasible to test more scenarios with differing error magnitudes and directions because of the large size of the test matrix and long simulation time for each run.

The along-track and cross-track errors are shown in Figure 12 as a function of the time elapsed in the basic horizontal trajectory scenario (as in Figure 7). The reported error values represent the mean and maximum errors in the single prediction made at that time in the scenario. For separation assurance, one is concerned with the maximum prediction error at any given time because a conflict may occur at any given time; for trajectory prediction accuracy one is concerned with how well the overall prediction compares to the actual trajectory. Figure 12 shows both these values. The salient characteristics of Figure 12 are the generally decreasing values of the errors as time increases, which is expected as the prediction horizon decreases, and the spikes in error that correspond to trajectory change points. These trajectory change points occur when CTAS increments the active waypoint and begins calculating a new trajectory before the aircraft reaches that waypoint. Changing the CTAS logic that selects the active waypoint will decrease the along-track error significantly. Also of interest in Figure 12 is the lower error experienced in the cross-track error than in the along-track error. This is a typical result that arises because the FMS normally is tracking a two-dimensional flight plan with a fixed airspeed, and not actively controlling the along-track distance to time. In the current concept of TBO, it is the automation's responsibility to predict that along-track distance to achieve correct knowledge of aircraft position. Employing RTA control in the FMS may improve the prediction and reduce the along-track error.

The prediction errors for a single auxiliary waypoint trajectory during cruise are shown in Figure 13. In this scenario there is a $10 \mathrm{kt}$ mismatch between the CTAS knowledge of winds and the actual wind field; however, comparison with Figure 12 shows that both along- and cross-track errors are actually smaller than the case where there exists perfect knowledge of the wind field. The reasons for this are uncertain, but they likely relate to logic
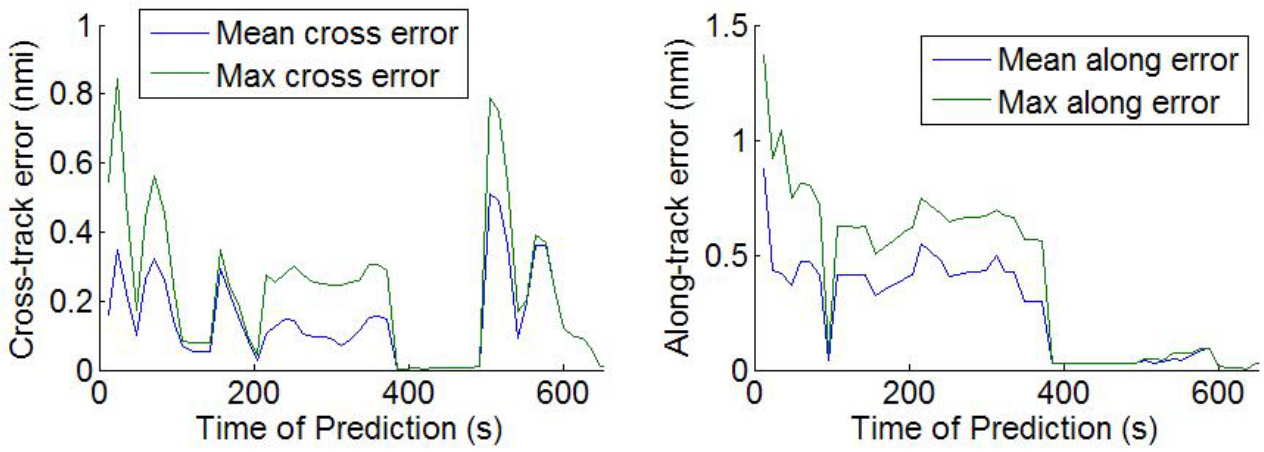

Figure 13. Along- and cross-track errors as a function of the time the prediction was made along the flight path. This scenario is for a single auxiliary waypoint clearance (see Figure 1) with a $10 \mathrm{kt}$ mismatch between simulator and CTAS winds. errors in CTAS' selection of the route that are coincidentally canceled by an unmodeled wind error (for example calculating the route to a downstream waypoint rather than a closer waypoint). This example shows that, circumstances where many uncertainties exist between air 


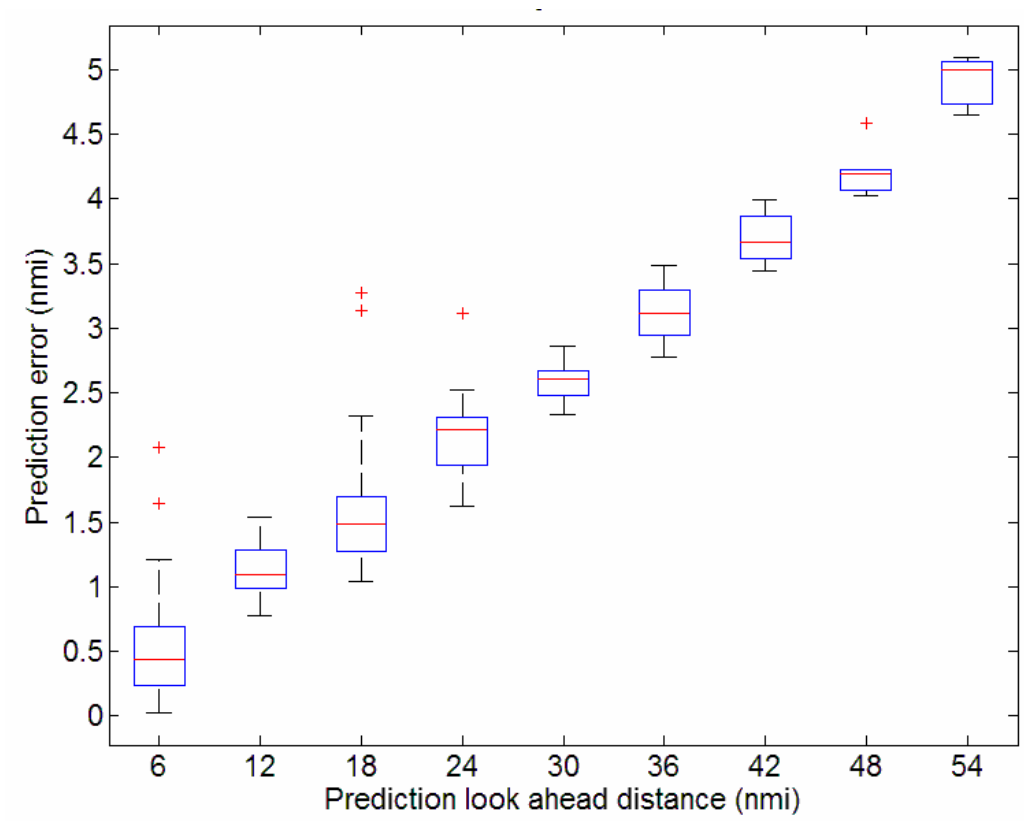

Figure 14. Horizontal distances (along- and cross-track) between actual aircraft position and the predicted position as a function of the prediction look-ahead time. Most of these errors are due to alongtrack errors. For each look-ahead time, the box and whisker plot conveys the median prediction error (red line in center of box), upper and lower quartiles (blue box), outliers (red ' + ' symbol), and the maximum extent of the data or 1.5 times the inter-quartile range, whichever is less (whiskers). These results are for a horizontal trajectory during climb (see Figure 1) with no mismatch between simulator and CTAS winds and perfect information on the aircraft's weight in the ground automation.

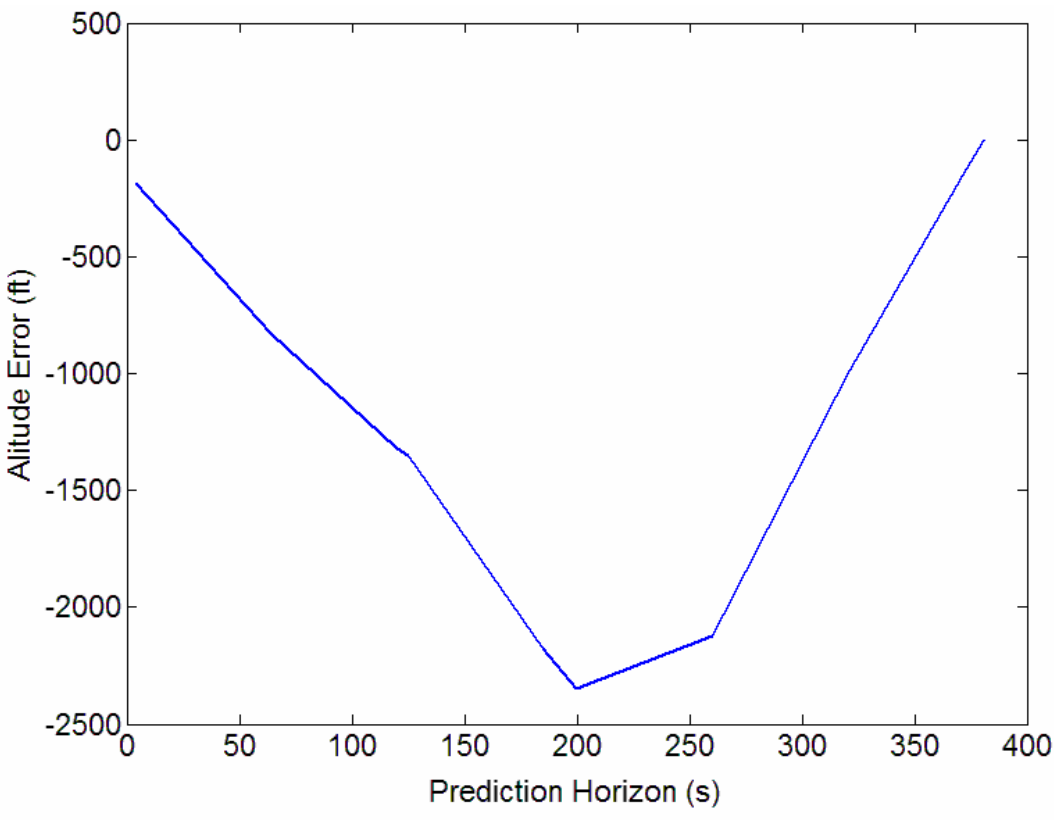

Figure 15. Altitude error as a function of look ahead time for the same clearance shown in Figure 14. and ground, an increase in the uncertainty of any given parameter may increase or decrease the accuracy of a prediction. The only way to consistently increase the accuracy of predictions is to decrease the uncertainty in many parameters at once by, for instance, using datalink to communicate aircraft weight, measured wind field, desired cruise speed, and other intent variables. ${ }^{28}$

The horizontal errors measured during a climb scenario with a single, lateral auxiliary waypoint as a function of prediction look-ahead distance are shown in Figure 14. A sample vertical prediction error is shown in Figure 15. The errors are significantly larger in vertical clearances than horizontal clearances because of the difficulty in predicting the climb rate of an aircraft even with weight information and the subsequent uncertainty in the location of the top of climb (TOC) point. Because an aircraft flies much more quickly in cruise rather than during climb, even a slight TOC error can lead to large along-track errors, which is the primary error source here. However, the magnitude of the error, particularly in the cross-track direction, is small enough that buffers in the separation detection function should safely account for the trajectory uncertainty. In addition, routines that identify an aircraft's current climb rate and then feed that data back into the prediction algorithm may increase the accuracy of such predictions without requiring an explicit measurement of the aircraft weight.

A summary of the mean and maximum errors in along-track, crosstrack and vertical directions as a function of scenario type and knowledge of aircraft weight is shown in Table 1 for the case in which CTAS has the same wind information as the simulator. The equivalent data for the case in which CTAS has a $10 \mathrm{kt}$ error in knowledge of the wind field are given in Table 2. Detailed study of those tables reveals several important 
conclusions, many related to the problem of reducing uncertainty in only one parameter of many and not achieving a corresponding increase in prediction accuracy. For example, a weight mismatch can increase the cross track error but decrease the along track error when wind mismatches between the simulator and ground automation are present. This result occurs because CTAS is predicting a higher climb speed than the 747 simulator is actually flying. This higher speed tends to offset the lower climb rate that CTAS predicts when it is overestimating the aircraft weight. When no weight mismatch is present, the higher predicted speed results in larger along-track errors. A similar effect is seen in the cruise scenarios - smaller errors result when a wind mismatch is present. This again occurs because CTAS's logic selects a route of flight slightly different from that planned by the FMS, and the unmodeled headwind happens by chance to correct some of the error. The complicated interaction of these variables shows that the prediction accuracy required for TBO is quite sensitive to the quantity and quality of information that is shared between air and ground automation systems, and that certain (possibly proprietary) information sources like aircraft weight may need to be shared for this concept to be feasible. Despite these issues, many of which can be corrected with improvements to the automation software, the analysis suggests that ground automation predictions are accurate enough in the horizontal trajectory for strategic trajectory-based separation assurance methods. The vertical trajectories will require further refinement of the prediction engine and additional data sources so that separation assurance can be done without unreasonably large uncertainty buffers.

Table 1. Track errors as a function of initial condition, trajectory type and error type (along, cross, vertical). The initial condition labeled "Climb, weight error" contains a mismatch between the actual aircraft weight and CTAS' assumption of the weight of approximately $20 \%$, a typical number. Winds in the simulator and in CTAS are identical. The error number before the slash is the mean error in prediction, the value after the slash is the maximum error among all the predictions for that scenario.

\begin{tabular}{|l|l|c|c|c|}
\hline Initial Condition & Trajectory Type & Along-track (nmi) & Cross-track (nmi) & Vertical (ft) \\
\hline \multirow{2}{*}{ Cruise } & Single Aux. Wpt. & $1.10 / 3.72$ & $0.43 / 0.67$ & - \\
\cline { 2 - 5 } & Direct-to & $0.86 / 3.10$ & $0.13 / 0.95$ & - \\
\hline \multirow{2}{*}{ Climb, 20\% weight error } & Single Aux. Wpt. & $2.45 / 10.52$ & $0.51 / 4.37$ & $1016 / 4943$ \\
\cline { 2 - 5 } & Interrupted Climb & $2.50 / 10.16$ & $0.40 / 4.25$ & - \\
\hline \multirow{2}{*}{ Climb, no weight error } & Single Aux. Wpt. & $3.07 / 12.74$ & $0.21 / 3.56$ & $279 / 1110$ \\
\cline { 2 - 5 } & Interrupted Climb & $4.27 / 12.17$ & $0.22 / 4.49$ & - \\
\hline
\end{tabular}

Table 2. This table contains the same information as that in Table 1, however in this case a wind error mismatch of $10 \mathrm{kt}$ exists between the simulator and CTAS.

\begin{tabular}{|l|l|c|c|c|}
\hline Initial Condition & Trajectory Type & Along-track (nmi) & Cross-track (nmi) & Vertical (ft) \\
\hline \multirow{2}{*}{ Cruise } & Single Aux. Wpt. & $0.25 / 1.37$ & $0.14 / 0.84$ & - \\
\cline { 2 - 5 } & Direct-to & $0.83 / 2.01$ & $0.07 / 0.87$ & - \\
\hline \multirow{2}{*}{ Climb, 20\% weight error } & Single Aux. Wpt. & $1.10 / 5.09$ & $0.49 / 3.27$ & $487 / 2347$ \\
\cline { 2 - 5 } & Interrupted Climb & $1.91 / 7.47$ & $0.34 / 2.93$ & - \\
\hline \multirow{2}{*}{ Climb, no weight error } & Single Aux. Wpt. & $3.38 / 12.06$ & $0.29 / 4.37$ & $361 / 1144$ \\
\cline { 2 - 5 } & Interrupted Climb & $3.78 / 10.67$ & $0.21 / 3.86$ & - \\
\hline
\end{tabular}

\section{Minimum WILCO Buffer Time}

The amount of time a controller must allow between sending a trajectory and expecting a response from the aircraft is still a matter of research for TBO. Average values have been reported for simpler clearances like altitude and speed changes on the order of 30 seconds $;{ }^{23,29}$ however, studies of operational datalink systems in Europe with more complex clearances have measured overall average response times above 60 seconds. ${ }^{30}$ Future simulations with operational flight crews will help determine the appropriate WILCO buffer time for each of these trajectory types, but a minimum value of 17 seconds was measured in this simulation. That minimum represents the average time necessary for the flight crew to manually press the required CDU buttons, wait for the FMS to load the trajectory, and confirm that the aircraft has switched to the new trajectory. It does not include the roundtrip message time, delays for the flight crew to begin responding to the message, time to consider the message before execution, and any delays in sending the WILCO back. While these limitations are quite severe, they do represent a lower limit on the amount of time required to execute a complex route clearance using current 747 FANS1 flight deck equipment. 


\section{Conclusions}

Current aircraft FMS (FANS1/A), existing datalink infrastructure (i.e., CPDLC), and prototype ground automation systems (CTAS) appear to be sufficient to support the technical implementation of trajectory-based operations. The strategic trajectory classes that are needed to support TBO can be implemented using the CPDLC message set, and actual aircraft trajectories based on FMS/autopilot execution of CPDLC messages appear to be consistent with the ground-generated trajectories upon which the CPDLC messages are based. Remaining questions to be answered in upcoming simulations are the procedural requirements of this concept, particularly how long the WILCO buffer must be between trajectory transmission and the start of the amended trajectory, the required amount of inter-sector and inter-center coordination to gain adequate benefits for users, the expected benefits to users in a mixed-equipage environment given the high fidelity datalink model constructed in the course of this work, and operations under failure and uncertainty modes.

\section{Appendix}

This is a potential generic flight deck procedure to be used when the controller issues a trajectory consisting of auxiliary waypoints with or without altitude constraints; any such constraints must be below the FMC cruise altitude. The aircraft may be climbing or level. The flight crew should be flying in LNAV/VNAV with the MCP dialed to the cruise altitude or higher. $\mathrm{PNF}=$ Pilot-non-flying. $\mathrm{PF}=$ Pilot flying.

1. ATC message chime is heard.

2. PNF or PF checks EICAS to confirm an ATC message was received and announces that fact.

3. PNF navigates to the ATC page on the CDU and selects the first unread message.

4. PF navigates to the LEGS page.

5. PNF reads the clearance aloud.

6. PNF selects the LOAD prompt once to bring the trajectory up on the nav display. (PNF should not ACCEPT the clearance until confirmation of its validity is established - see step 12.)

7. PF scans for discontinuities in the new flight plan in legs page, announcing their presence if they do occur. There are two procedural options from this point (only one of which would be asked of the flight crew on any particular simulation run):

a. Option 1: Do not immediately reject the message. Contact the controller over voice and get further instructions. The controller has three options:

i. Tell the crew to reject the message and wait for another datalink clearance.

ii. Tell the crew to proceed direct to the second auxiliary waypoint (the first waypoint after the WILCO buffer point).

iii. Issue (tactical) instructions as they would under today's operations.

b. Option 2: Immediately reject the message. Contact the controller over voice and get further instructions. The controller has two options:

i. Tell the crew to wait for another datalink clearance.

ii. Issue (tactical) instructions as they would under today's operations.

8. PF scans the trajectory on the legs page to determine whether any altitude constraints are associated with the new waypoints. (This should only occur if the aircraft is currently climbing.) PF reads the constraint aloud if one is present.

9. PF scans the entire amended trajectory on the nav display to ensure it is valid and feasible.

10. PNF adjusts the nav display range so that the distance to the WILCO buffer point is clearly visible (20 or $40 \mathrm{~nm}$ range) and monitors that point making sure the aircraft does not pass it before the trajectory is executed. If the aircraft does pass the WILCO point before the crew determines that the trajectory is good they must contact the controller over the voice channel (do not REJECT the message). Controller has the same three options that are listed under 7a, i-iii.

11. When each crew member is satisfied with the new trajectory, they announce that the trajectory is good. If they find the new trajectory unsatisfactory they may reject it and should provide a reason.

12. PNF selects the ACCEPT prompt, announcing "accepting the message."

13. PF selects the EXECUTE prompt, announcing "executing the trajectory."

14. PNF selects the WILCO prompt (may also be labeled "Response Send").

15. PF/PNF may optionally dial the new heading into the MCP, but should remain on LNAV. 


\section{Acknowledgements}

E. Mueller would like to thank Dave McNally and Sandy Lozito for providing significant technical expertise in planning this investigation and Tony Wang for writing most of the new software in the ground automation system and datalink emulator. He also thanks the many people who contributed to building the simulation capabilities described in this paper, Debbi Ballinger, Diane Carpenter, Srba Jovic, Rod Ketchum, Ron Lehmer, Terry Rager, and Ghislain Saillant, along with the researchers who provided recommendations on the content of the investigation: Lewis Bailey, Rich Coppenbarger, Tom Holford, Jim Mast, Rob Mead and Tom Prevot.

\section{References}

1 “Next Generation Air Transportation System Integrated Plan,” Department of Transportation, Dec. 12, 2004.

${ }^{2}$ Prevot, T., Palmer, E., Crane, B., "Flight Crew Support for Automated Negotiation of Descent and Arrival Clearances," Proceedings of the Ninth International Symposium on Aviation Psychology, Vol. 1, Columbus, OH, 1997.

3 Wichman, K., Lindberg, L., Kirchert, L., Bleeker, O., "Four-Dimensional Trajectory Based Air Traffic Management," AIAA Guidance, Navigation and Control Conference, Vol. 1, Washington, D.C., Aug., 2004.

${ }^{4}$ Wichman, K., Carlsson, G., Lindberg, L., "Flight Trials: 'Runway-to-Runway' Required Time of Arrival Evaluations for Time-Based ATM Environment - Final Results," AIAA Guidance, Navigation and Control Conference, Vol. 1, Washington, D.C., Aug., 2002.

${ }^{5}$ Denery, D. G. and Erzberger, H., "The Center-TRACON Automation System: Simulation and Field Testing," NASA TM-110366, August, 1995.

${ }^{6}$ Sullivan, B., Panda, R., Soukup, P., "Implementing FANS in the NASA 747-400 Flight Simulator for Airspace Operational Research (Future Air Navigation System)," AIAA Flight Simulation Technologies Conference, Vol. 1, Washington, D.C., 1996.

${ }^{7}$ Bolczak, R., "Operational Evolution of an Integrated URET/CPDLC Capability," $21^{\text {st }}$ Digital Avionics Systems Conference, Vol. 1, Irvine, CA, 2002.

${ }^{8}$ Celio, J., "CPDLC Integration with URET - A Concept for Improving Airspace User Services," $20^{\text {th }}$ Digital Avionics Systems Conference, Vol. 1, Daytona Beach, FL, October, 2001.

${ }^{9}$ Williams, D., Green, S., "Piloted Simulation of an Air-Ground Profile Negotiation Process in a Time-Based Air Traffic Control Environment," NASA TM-107748, April, 1993.

${ }^{10}$ Green, S., and Vivona, R., "Field Evaluation of Descent Advisor Trajectory Prediction Accuracy," AIAA Guidance, Navigation and Control Conference, Vol. 1, Washington, D.C., July, 1996.

${ }^{11}$ Oseguera, R., Williams, D., "Flight Evaluation of the CTAS Descent Advisor Trajectory Prediction," American Control Conference, Seattle, Washington, June 1995.

${ }^{12}$ Prevot, T., Shelden, S., Palmer, E., Johnson, W., Battiste V., and Smith, N., "Distributed Air/Ground Traffic Management Simulation: Results, Progress and Plans," AIAA Modeling and Simulation Technologies Conference and Exhibit, Vol. 1, Washington, D.C., Aug., 2003.

${ }^{13}$ T. Prevot, E. Palmer, N. Smith and T. Callantine., "A Multi-Fidelity Simulation Environment for Human-In-TheLoop Studies of Distributed Air Ground Traffic Management," AIAA Modeling and Simulation Technologies Conference and Exhibit, Washington, D.C., Aug., 2002.

14 Oseguera-Lohr, R., Williams, D., "Evaluation of Trajectory Errors in an Automated Terminal-Area Environment," NASA/TM-2003-212174, April, 2003.

${ }^{15}$ Smith, N., Lee, P., Prevot, T., Mercer, J., Palmer, E., Battiste, V., and Johnson, W., "A Human-in-the-Loop Evaluation of Air-Ground Trajectory Negotiation," AIAA Aviation Technology, Integration and Operations Forum, Vol. 1, Sep., 2004.

${ }^{16}$ Mackintosh, M., Lozito, S., McGann, A., Logsdon, E., "Designing Procedures for Controller-Pilot Data Link Communication: Effects of Textual Data Link on Information Transfer," AIAA World Aviation Conference, Vol. 1, Washington, D.C., October, 1999.

${ }^{17}$ Lozito, S., Verma, S., Martin, L., Dunbar, M., McGann, A., "The Impact of Voice, Data Link, and Mixed Air Traffic Control Environments on Flight Deck Procedures," Air Traffic Control Quarterly, Vol. 11, No. 4, 2003, pp. 293-310.

${ }^{18}$ Crane, B., Prevot, T., Palmer, E., "Flight Crew Factors for CTAS/FMS Integration in the Terminal Area," NASA TM-2000-209607, 2000.

${ }^{19}$ Mumaw, R., Sarter, N., Wickens, C., "Analysis of Pilot's Monitoring and Performance on an Automated Flight Deck," $11^{\text {th }}$ International Symposium on Aviation Psychology, Columbus, Ohio, 2001. 
${ }^{20}$ Smith, N., Brown, J., Polson, P., Moses, J., “An Assessment of Flight Crew Experiences with FANS-1 ControllerPilot Data Link Communication in the South Pacific," $4^{\text {th }}$ USA/Europe Air Traffic Management R\&D Seminar, Santa Fe, NM, December 2001.

${ }^{21}$ McNally, D., Gong, C., "Concept and Laboratory Analysis of Trajectory Based Automation for Separation Assurance," Air Traffic Control Quarterly, Vol. 15, No. 1, 2007.

${ }^{22}$ Paielli, R., Erzberger, H., "Improved Conflict Detection for Reducing Operational Errors in Air Traffic Control," AIAA 2004-6392, AIAA Aircraft Technology, Integration and Operations Forum, Vol. 1, Washington, D.C., Sep., 2004.

${ }^{23}$ Bolczak, R., Gonda, J., Saumsiegel, W., Tornese, R., "Controller-Pilot Data Link Communications Build 1 ValueAdded Services," 23 ${ }^{\text {rd }}$ Digital Avionics Systems Conference, Salt Lake City, October, 2004.

${ }^{24}$ Bennett, M., Knorr, D., Rakas, J., "Economic Benefits of Increased En Route Sector Capacity from ControllerPilot Data Link Communications," 83 ${ }^{\text {rd }}$ TRB Annual Meeting, Transportation Research Record, 2004.

${ }^{25}$ Hasan, H., Leiden, K., Mondoloni, S., Kozarsky, D., Green, S., “An Initial Benefits Assessment of Distributed Air/Ground Traffic Management Concept Elements," AIAA Aviation Technology, Integration, and Operations Forum, Vol. 1, Nov., 2003.

${ }^{26}$ Williams, D., Green, S., “Airborne Four-Dimensional Flight Management in a Time-Based Air Traffic Control Environment,” NASA Technical Memorandum 4249, March 1991.

${ }^{27}$ Computer Sciences Corporation, "Life-Cycle Cost/Benefit Assessments of DAG-TM Concept Elements 5, 6 and 11. Version 4.0, Final Report," Air Traffic Management System Development and Integration, NASA Ames Research Center, Doc ID CSC/ATMSDI/CTO-08-10/040630, June 2004.

${ }^{28}$ Coppenbarger, R., Mead, R., Sweet, D., "Field Evaluation of the Tailored Arrivals Concept for Datalink-Enabled Continuous Descent Approach," AIAA Aviation Technology, Integration and Operations Conference, Belfast, U.K., Sep., 2007.

${ }^{29}$ Prevot, T., Lee, P., Martin, L., Mercer, J., Palmer, E., Smith, N., “Tools for Trajectory-Based Air Traffic Control and Multi Sector Planning," HCI-Aero'06, Seattle, WA, Sep. 2006.

30 “PETAL Newsletter,” PETAL-to-LINK Programme, Eurocontrol, Vol. 4, No. 2, Apr. 2002. 